\title{
AN EXTENDED METHOD FOR STUDING CYLINDRIC STRUCTURE BY USING ITERATIVE METHOD
}

\author{
Beldi, S., R. Bedira and A. Gharsallah \\ Unité de Recherche Circuits et Systèmes Electroniques HF, \\ Faculté Des Sciences de Tunis, Campus Universitaire Tunis EL-Manar, 2092, Tunisie
}

Received 2013-11-01; Revised 2013-11-26; Accepted 2013-06-26

\begin{abstract}
The method iterative Wave Concept Iterative method Procedure (WCIP) avoids the undesired phenomenon of unbounded operators; relations between currents and fields, obtained using unbounded impedance operators, are transposed to relations between waves, supplied by bounded scattering operators.
\end{abstract}

Keywords: Electromagnetic Modeling, Iterative Method, The Concept of Waves, Cylindrical Antennas

\section{INTRODUCTION}

The scattering of electromagnetic waves in free space has been the subject of numerous studies, which derive several numerical methods such as finite element method, the method of moments, these methods are limited in their applications and they require enough memory space important. The iterative method (W.C.I.P) of modeling will guarantee the speed time of calculation, the precision of the results obtained, the insurance to obtain the convergence and the adaptability with all types of structures. This method is based on the determination of the operators of diffraction in the spectral and space domain which call the operators of admittances or impedances. These operators of diffraction can bind the tangential waves to the interfaces of the considered structure. The method for electromagnetic modeling is generally based on a formulation of the wave; it involves operators of impedances or admittances. The concept wave has advantages over methods electromagnetic modeling, such as insurance of convergence and the gain of computation time (Beldi et al., 2011; Azizi et al., 2013). With the diffraction operator is assured of convergence (these operators are bounded). On the hand, operators of diffraction space are always defined on the whole interface, but this is not the case for operators of admittance or impedance that matches it (Baudrand and Wane, 2009). In a comprehensive analysis of the field and from sources of electric and magnetic currents, we use the Green operators (Latrach et al., 2009). In this kind of analysis, the operators of impedance or admittance have the disadvantage of being unlimited and therefore the digital convergence is not always guaranteed. The integral formulation in a transmission line with the method of line TLM transmission (Glaoui et al., 2009) is usually defined in the time domain but is expressed in spectral domain (Pasalic et al., 2001; Harizi and Gharsallah, 2012). This spectral representation is the basis of a method called the iterative process of design Waves (WCIP).

\section{ITERATIVE CONCEPT}

The modeling of problem in the case with or without source, they are two types of sources is current source of electric field is imposed in the problem to be studied. So they appear different systems which are deducted directly from the expressions (1) and (2). They include either a spatial wave source (3), a modal wave source (4) Equation 1 to 4:

Corresponding Author: Beldi, S., Unité de Recherche Circuits et Systèmes Electroniques HF, Faculté des Sciences de Tunis, Campus Universitaire Tunis EL-Manar, 2092, Tunisie 


$$
\left\{\begin{array}{c}
\vec{A}=\hat{S} \vec{B} \\
\vec{B}=\hat{\Gamma} \vec{A}+\vec{B}_{0}
\end{array}\right.
$$

With:

$$
\vec{A}=\left(\begin{array}{l}
\vec{A}_{1} \\
\vec{A}_{2}
\end{array}\right)
$$

$$
\vec{B}=\left(\begin{array}{l}
\vec{B}_{1} \\
\vec{B}_{2}
\end{array}\right)
$$

\section{With:}

Spatial wave source:

$$
\vec{A}_{0}=\left(\begin{array}{l}
\vec{A}_{01} \\
\vec{A}_{02}
\end{array}\right)
$$

Modal wave source:

$$
\vec{B}_{0}=\left(\begin{array}{l}
\vec{B}_{01} \\
\vec{B}_{02}
\end{array}\right)
$$

In the formulation waves with diffraction operators are spatial or model, when the convergence is ensured using diffraction bounded operators which allow us to have a low computation time. This method allows modeling any structure. To solve this system, we use the iterative process in both cases the excitation modal or spatial.

\section{FIRST DIFFRACTION BY PALLETS CYLINDRIC STRUCTURE}

\subsection{Design}

Consider a structure of cylindrical geometry made of two materials: Metal and dielectric perfect (Fig. 1). To study the phenomenon of diffraction by such a structure, the target is bombarded by a plane wave TM (z) at normal incidence with an angle of incidence $\theta_{\text {inc }}=-90^{\circ}$. Solving the problem is based on the wording on the air in the cylindrical coordinate system (Raveu, 2003).

\subsection{Formulationin the Case of Cylindrical Pallets}

There are two areas operators diffraction to study, one in field space and the other in field modal. The operator of diffraction in the space defined on the cylinder by a matrix $\hat{s}$ of diffraction according to Equation 5:

$\hat{S}=\left(\begin{array}{cc}-\hat{\mathrm{H}}_{\mathrm{Dm}} & \hat{\mathrm{H}}_{\mathrm{Dd}} \\ \hat{\mathrm{H}}_{\mathrm{Dd}} & -\hat{\mathrm{H}}_{\mathrm{Dm}}\end{array}\right)$

With:

$\hat{\mathrm{H}}_{\mathrm{Dm}}=$ Indicates the field metal

$\hat{\mathrm{H}}_{\mathrm{Dd}}=$ Indicates the field dielectric

In this case we have two operators in modal diffraction. The operator describes the external diffraction reflection coefficient outside the cylindrical pallets. In this case, there was an invariant in $\mathrm{z}, \mathrm{k}_{\mathrm{m}}=0$ then $\mathrm{k}_{\mathrm{\rho}}=\mathrm{k}_{0}$ then separation into TE and TM modes is accurate.

In the cylindrical structure of the pallets, the source is a transverse magnetic plane wave along the $\mathrm{z}$ direction and the structure has a z-invariance. So we consider only the following components in the $\theta$. Therefore, there is no periodicity along $\mathrm{z}$; the distance of the walls is not involved in regular problem solving (Houaneb et al., 2011).

We deduce wave source in modal $\mathrm{B}_{0}^{\mathrm{z}}$ from the field incident $\mathrm{TM}(\mathrm{z})$ mode $\mathrm{E}_{\mathrm{z}}^{\mathrm{inc}}$ expressed in cylindrical coordinates Equation 6:

$E_{z}^{i n c}=E_{0} \sum_{n=0}^{+\infty}\left(2-\delta_{n 0}\right) i^{-n} J_{n}\left(k_{0} \rho\right) \cos \left[n\left(\theta-\theta_{i n c}\right)\right]$

With:

$$
\delta_{n 0}=\left\{\begin{array}{l}
1 \rightarrow n=0 \\
0 \rightarrow n \neq 0
\end{array} k_{0}=w \sqrt{\mu_{0} \varepsilon_{0}}\right.
$$

After the determination of diffraction coefficients in modal and spatial structure can be modeled by a diagram as shown in Fig. 2.

After expressing all sizes needed to produce the waves, we can apply the iterative process corresponds. Knowing that the source is defined in modal. The equations governing the iterative process are written according to the following Equation 7:

$$
\begin{aligned}
& \left\{\begin{array}{l}
B_{1}^{z}=\hat{\Gamma}_{\text {int }}^{z} A_{1}^{z} \\
B_{2}^{z}=\hat{\Gamma}_{e x t}^{z} A_{2}^{z}+B_{0}^{z}
\end{array}\right. \\
& \left(\begin{array}{l}
A_{1}^{z} \\
A_{2}^{z}
\end{array}\right)=(\hat{S})\left(\begin{array}{l}
B_{1}^{z} \\
B_{2}^{z}
\end{array}\right)
\end{aligned}
$$




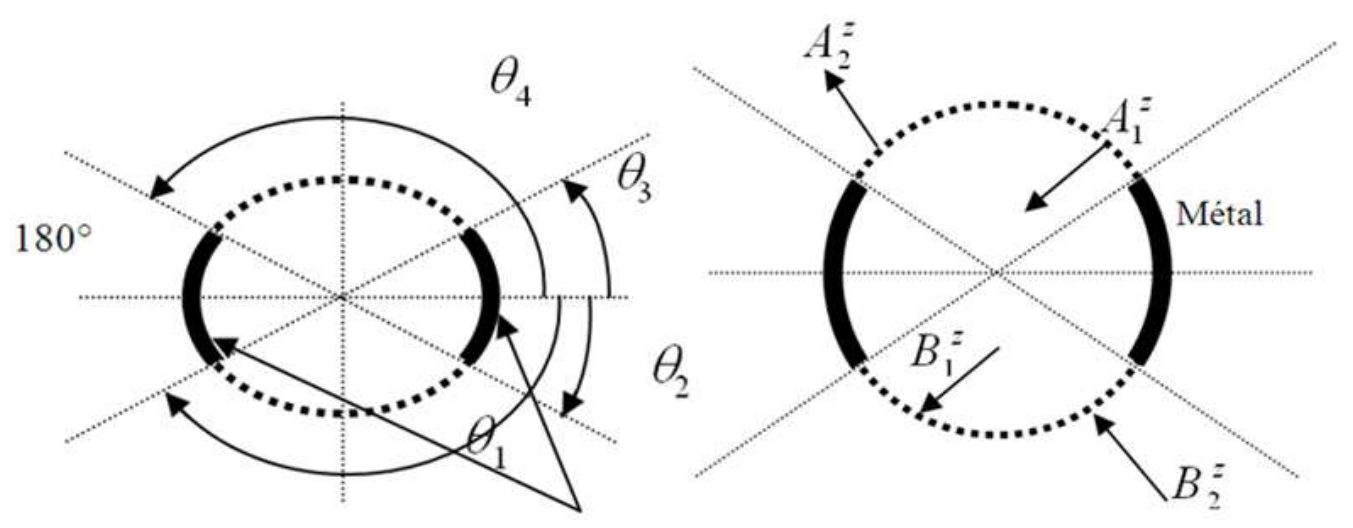

Fig. 1. (a) pallets cylindrical section (b) Definition of waves

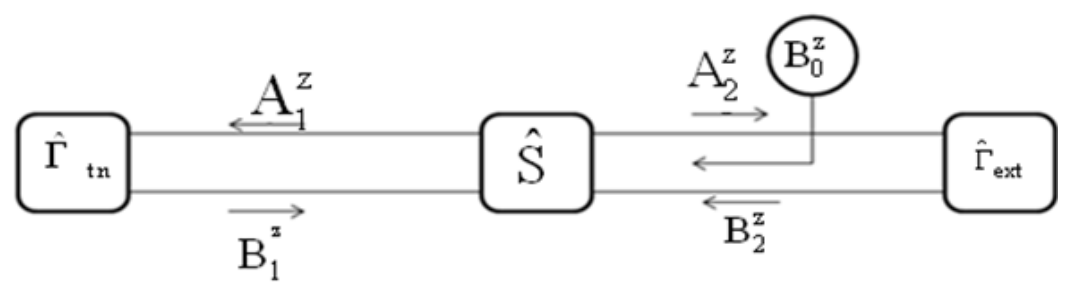

Fig. 2. Modélisation de la structure avec la formulation en ondes

Once convergence is reached, we can calculate the electromagnetic quantities diffracted by the structure. We defined the module electrical fields $|\mathrm{Ez}|$ normalized electric field incidents according to Equation 8, with $K_{0} R_{1}=$ $2 \pi$ is a number of mode $N_{\theta}=64$. We then analyze the variation of the field module normalized the dimensions of metal pallets of cylindrical geometry Equation 8:

$E_{z}(\theta)=\sqrt{Z_{0}} \frac{A_{1}^{z}(\theta)+B_{1}^{z}(\theta)}{E_{z}^{i n c}(\theta)}$

\subsection{Results}

The coefficients of diffraction modal are show in the Fig. 3 and 4.

Figure 3 shows the variation of the real part of scattering coefficient with a number of external modes in $\theta$ with $N=64$. This theory is based on what is developed for development of the external diffraction coefficient except that in this example has invariance in $\mathrm{z}$. The internal scattering operator describes the reflection coefficient inside the cylindrical structure confined between the pallets.

Figure 4 also shows the variation of the real part of the reflection coefficient with a number of internal modes of $N_{\theta}=64$.
We apply the iterative process in this example is attained after 203 iterations for convergence $|\mathrm{Ez}|$ shows in the Fig. 5.

First simulation:

$$
\left\{\theta_{1}, \theta_{2}, \theta_{3}, \theta_{4}\right\}=\left\{-155^{\circ},-25^{\circ}, 25^{\circ}, 155^{\circ}\right\}
$$

Second simulation:

$$
\left\{\theta_{1}, \theta_{2}, \theta_{3}, \theta_{4}\right\}=\left\{-120^{\circ},-60^{\circ}, 60^{\circ}, 120^{\circ}\right\}
$$

Third simulation:

$$
\left\{\theta_{1}, \theta_{2}, \theta_{3}, \theta_{4}\right\}=\left\{-105^{\circ},-75^{\circ}, 75^{\circ}, 105^{\circ}\right\}
$$

Note that the variations of the electric field at the pallet are zero against it by a variation of the field at the cylindrical openings of about $1.2 \mathrm{~V} / \mathrm{m}$ show in Fig. 6. In this example, the wording on the waves has been successfully applied to the diffraction of a plane wave of TM (z) for cylindrical conductive pallets of various sizes. In this modeling diffraction modal operators are used externally and internally in the case of invariance in z. At an angle of incidence of the wave at $-90^{\circ}$ are the maximum variations of electric fields on the slots. 
Beldi, S. et al. / American Journal of Applied Sciences 11 (8): 1426-1435, 2014

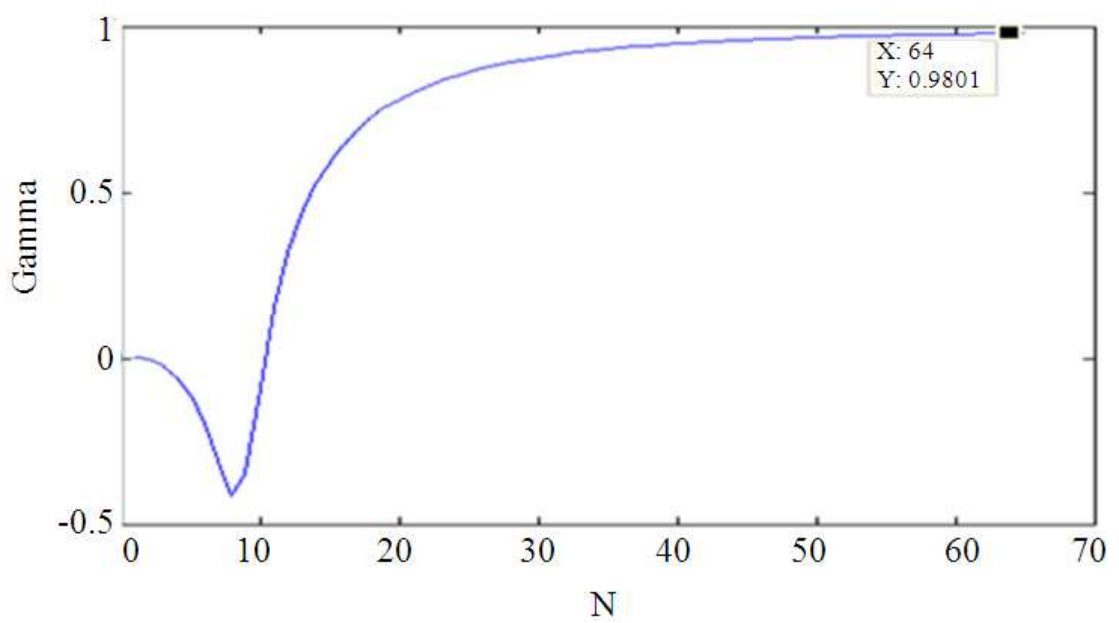

Fig. 3. Real part of Gamma many external modes of $N \theta=64$

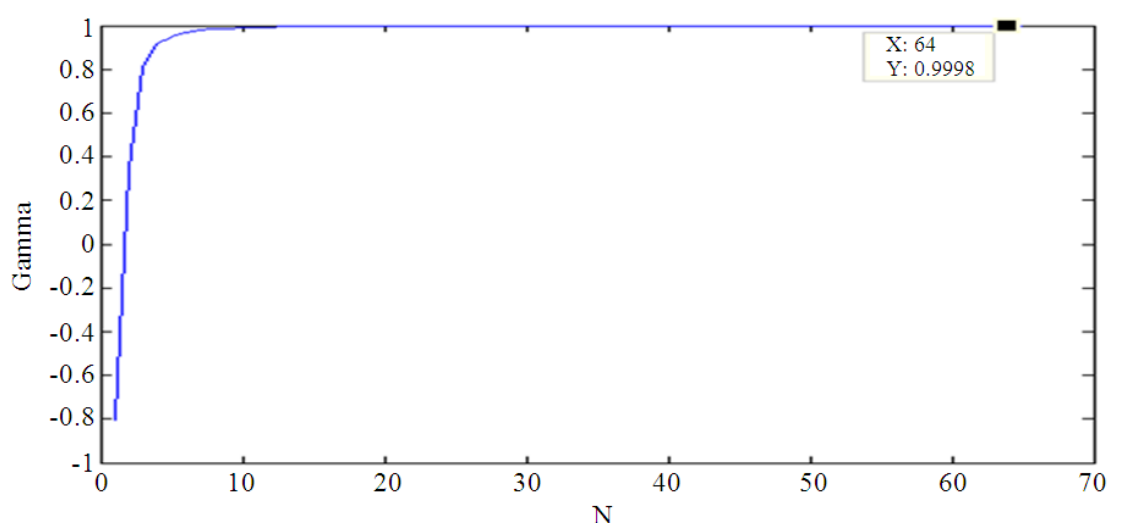

Fig. 4. Real part of Gamma many internal modes of $N \theta=64$

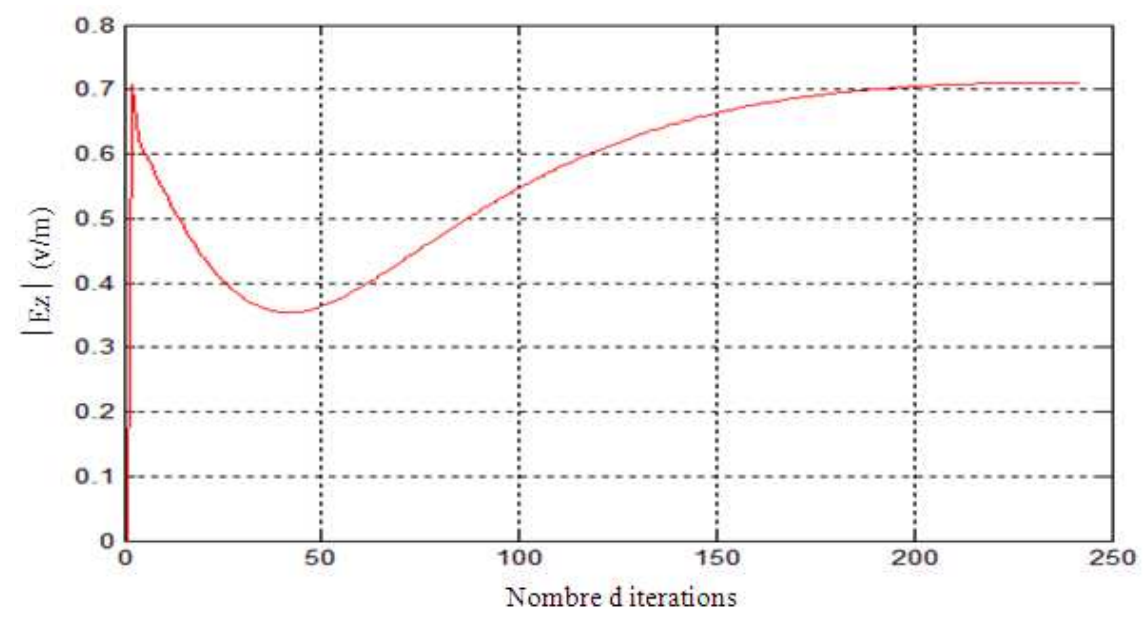

Fig. 5. Convergence $|E z|$ by the number of iterations 


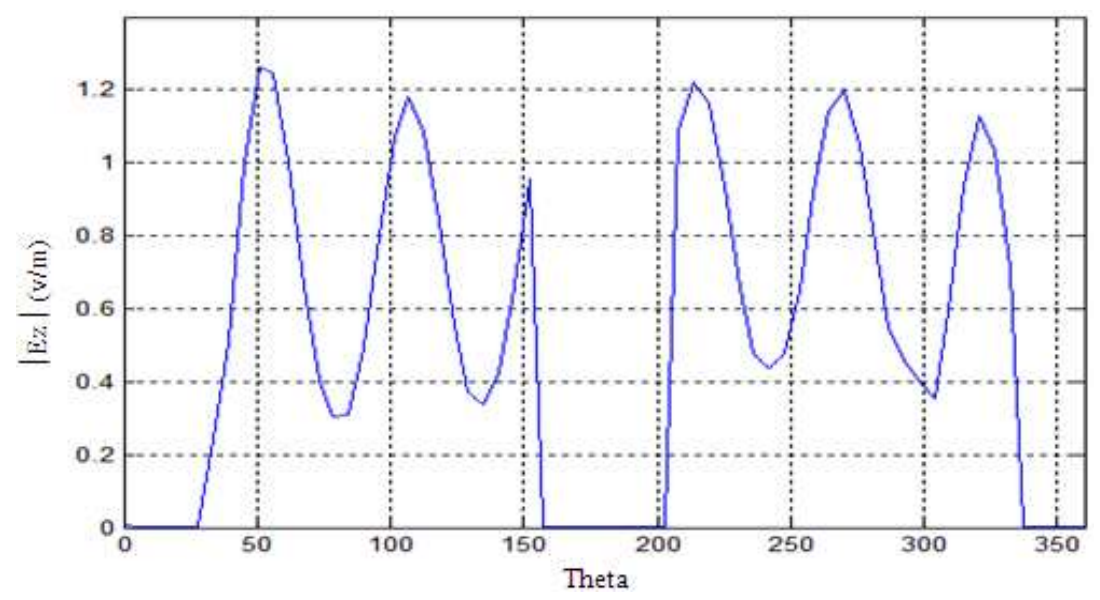

Fig. 6. Normalized fields $|E z|$ on the surface of diffraction for: $\left\{\theta_{1}, \theta_{2}, \theta_{3}, \theta_{4}\right\}=\left\{-155^{\circ},-25^{\circ}, 25^{\circ}, 155^{\circ}\right\}$

\section{SECAND STRUCTURES OF CYLINDRICAL SLOT ANTENNAS}

\subsection{Design}

In this section, the coupling between axial slots Fig. (9a) and radial slots Fig. (9b), on a perfectly conducting cylinder is evaluated based on separation distances angular $\theta_{0}$ and azimuthally $\mathrm{h}_{0}$ at the frequency $9 \mathrm{GHz}$. This example shows only a surface perfectly metallic cylinder $\mathrm{C}$ of infinite length. The equivalent circuit consists of two operators: An operator which defines the surface $\mathrm{C}$ and another that defines the space surrounding the surface $\mathrm{C}$. At the surface of a perfectly conducting cylinder of infinite length in the $\mathrm{z}$ direction of radius $\mathrm{r}=5.057 \mathrm{~cm}$, there are two types of radiating slots rectangular (Fig. 9):

- In the case of axial slots (Fig. 7) $\mathrm{L}_{\theta}=1.016$ and $\mathrm{L}_{\mathrm{z}}=$ $2.032 \mathrm{~cm}$, the field $\mathrm{E}_{\theta}$ issued, only the TE01 mode at a frequency $9 \mathrm{GHz}$

- In the case of radial slots (Fig. 8) $\mathrm{L}_{\theta}=1.016$ and $\mathrm{L}_{\mathrm{z}}=$ $2.032 \mathrm{~cm}$, the field $\mathrm{E}_{\theta}$ issued, only the TE01 mode at a frequency $9 \mathrm{GHz}$

\subsection{Formulation}

The transmission coefficient $S_{12}$ between the two waveguides is determined for a distance $h_{0}$ and several azimuthally angular distances $\theta_{0}$. This transmission coefficient is derived from the projection on the wave propagation modes guide Equation 9:

$S_{12}=\frac{\left\langle B_{i} \mid f_{0}^{\prime}\right\rangle}{\left\langle A_{i} \mid f_{0}\right\rangle}$
With $\mathrm{i}$ denoting the component of the wave that occurs in the coupling $(\theta, z)$.

For axial slots, means the generating function of $\mathrm{TE}_{01}$ mode in the direction $\theta$ at emissions guide, means the same generating function as a guide at the same receiving and generating functions associated to the radial openings. Incoming and outgoing waves in the slots are projected on the basis of spectral modes of the waveguide such as Equation 10 and 11:

$\left.B_{i}\right|_{f e}(x, y)=\sum_{n} \sum_{m} b_{n m f e}^{T E} g_{n m}^{T E \alpha}+b_{n m f e}^{T M} g_{n m}^{T M \alpha}$

$\left.A_{i}\right|_{f e}(x, y)=\sum_{n} \sum_{m} a_{n m f e}^{T E} g_{n m}^{T E \alpha}+a_{n m f e}^{T M} g_{n m}^{T M \alpha}$

$=\sum_{n} \sum_{m} \gamma_{n m}^{T E} b_{n m f e}^{T E} g_{n m}^{T E x}+\gamma_{n m}^{T M} b_{n m f e}^{T M} g_{n m}^{T M \alpha}$

Where Equation 12:

$$
\left.\begin{array}{l}
g_{n m}^{T E x}=A_{n m}^{T E x} \cos \left[\frac{n \cdot \pi}{L_{\theta}}\left(a \theta-\frac{L_{\theta}}{2}\right)\right] \sin \left[\frac{m \cdot \pi}{L_{z}}\left(z-\frac{L_{z}}{2}\right)\right] \\
g_{n m}^{T E y}=A_{n m}^{T E y} \cos \left[\frac{n \cdot \pi}{L_{\theta}}\left(a \theta-\frac{L_{\theta}}{2}\right)\right] \sin \left[\frac{m \cdot \pi}{L_{z}}\left(z-\frac{L_{z}}{2}\right)\right] \\
g_{n m}^{T M y}=A_{n m}^{T M y} \cos \left[\frac{n \cdot \pi}{L_{\theta}}\left(a \theta-\frac{L_{\theta}}{2}\right)\right] \sin \left[\frac{m \cdot \pi}{L_{z}}\left(z-\frac{L_{z}}{2}\right)\right] \\
g_{n m}^{T M x}=A_{n m}^{T M x} \cos \left[\frac{n \cdot \pi}{L_{\theta}}\left(a \theta-\frac{L_{\theta}}{2}\right)\right] \sin \left[\frac{m \cdot \pi}{L_{z}}\left(z-\frac{L_{z}}{2}\right)\right]
\end{array}\right\}
$$


$\mathrm{g}_{\mathrm{nm}}^{\text {TEx }}, \mathrm{g}_{\mathrm{nm}}^{\text {TEy }}, \mathrm{g}_{\mathrm{nm}}^{\mathrm{TMx}}$ et $\mathrm{g}_{\mathrm{nm}}^{\mathrm{TMx}}$ are the proper functions of the modal basis of the guides in the Cartesian coordinate system, we consider $\mathrm{y}$ that it corresponds to the $\mathrm{z}$ axis and $\mathrm{x}$ axis $\mathrm{r}_{\theta}$, the curvature being neglected as indicated.

The normalization coefficients of the proper functions of the modal basis are Equation 13 to 15 :

$$
\begin{gathered}
A_{n m}^{T E x}=\sqrt{\frac{2 \tau_{n m}}{L_{\theta} L_{z}}} \cdot \frac{m}{L_{z} \sqrt{\left(\frac{n}{L_{\theta}}\right)^{2}+\left(\frac{m}{L_{z}}\right)^{2}}}=-A_{n m}^{T M y} \\
A_{n m}^{T E y}=\sqrt{\frac{2 \tau_{n m}}{L_{\theta} L_{z}}} \cdot \frac{m}{L_{z} \sqrt{\left(\frac{n}{L_{\theta}}\right)^{2}+\left(\frac{m}{L_{z}}\right)^{2}}}=A_{n m}^{T M x} \\
\tau_{\mathrm{nm}}=\left\{\begin{array}{l}
2 \operatorname{Sin} \neq 0 \text { et } \mathrm{m} \neq 0 \\
1 \text { Sin }=0 \text { et } \mathrm{m}=0
\end{array}\right.
\end{gathered}
$$

The structure consists of a perfectly conducting cylinder with two rectangular slots whose radiating operator diffraction space is special. The boundary conditions on the metal pixels remain unchanged. By cons at the junction of the rectangular waveguide and cylindrical perfectly conducting surface, the boundary conditions must take into account the reaction of the guide, this is achieved through the pixel mode. As these rectangular guides have metal walls, the last are electric walls. The TE and TM modes which are developed in the Cartesian system can be separated. Subsequently, we decompose the wave at the slots of the guides on a space station, which will consist of unit steps on the pixels rectangular guides along the directions $\theta$ and $\mathrm{z}$. The boundary conditions on the perfectly conducting cylinder are taken into account by the operator of diffraction space by writing matrix as Equation 16:

$$
\hat{\mathrm{S}}=\left(\begin{array}{cc}
-\hat{\mathrm{H}}_{\mathrm{m}}+\hat{\mathrm{H}}_{\mathrm{fe}}\left[\hat{\chi}_{\theta}\right]_{\theta \mathrm{ozo}} & 0 \\
0 & -\hat{\mathrm{H}}_{\mathrm{m}}+\hat{\mathrm{H}}_{\mathrm{fe}}\left[\hat{\chi}_{\mathrm{z}}\right]_{\theta \mathrm{ozo}}
\end{array}\right)
$$

where, $\hat{\mathrm{H}}_{\mathrm{fe}}$ denotes the projector onto the pixels of the guide.

$\hat{\mathrm{H}}_{\mathrm{m}} \cup \hat{\mathrm{H}}_{\mathrm{fe}}$ Represents the entire surface of the cylinder. $\left[\hat{\chi}_{\theta}\right]_{\theta \text { ozo }}$ and $\left[\hat{\chi}_{z}\right]_{\theta \text { ozo }}$ represent the modal response guides positioned at the pixel level, in $\theta_{0}$ and $\mathrm{h}_{0}$ Equation 17 and 18:

$$
\begin{aligned}
& {\left[\hat{\chi}_{\theta}\right]_{\theta 0 z o}=\sum_{\mathrm{k}} \sum_{\mathrm{l}}\left\langle\mathrm{q}_{\theta \mathrm{ozo}}\left|\mathrm{g}_{\mathrm{pq}}^{\mathrm{TEz}}\right| \gamma_{\mathrm{pq}}^{\mathrm{TE}}\left\langle\mathrm{g}_{\mathrm{pq}}^{\mathrm{TEz}} \mid \mathrm{q}_{\mathrm{kl}}\right\rangle\right.} \\
& +\sum_{\mathrm{k}} \sum_{\mathrm{l}}\left\langle\mathrm{q}_{\theta \mathrm{ozo}} \mid \mathrm{g}_{\mathrm{pq}}^{\mathrm{TMz}}\right\rangle \gamma_{\mathrm{pq}}^{\mathrm{TM}}\left\langle\mathrm{g}_{\mathrm{pq}}^{\mathrm{TMz}} \mid \mathrm{q}_{\mathrm{kl}}\right\rangle
\end{aligned}
$$

$$
\begin{aligned}
& {\left[\hat{\chi}_{\mathrm{z}}\right]_{\theta 0 \mathrm{zo}}=\sum_{\mathrm{k}} \sum_{\mathrm{l}}\left\langle\mathrm{p}_{\theta 0 \mathrm{ozo}}\left|\mathrm{g}_{\mathrm{pq}}^{\mathrm{TEz}}\right| \gamma_{\mathrm{pq}}^{\mathrm{TE}}\left\langle\mathrm{g}_{\mathrm{pq}}^{\mathrm{TEz}} \mid \mathrm{p}_{\mathrm{kl}}\right\rangle\right.} \\
& +\sum_{\mathrm{k}} \sum_{1}\left\langle\mathrm{p}_{\theta 0 \mathrm{ozo}} \mid \mathrm{g}_{\mathrm{pq}}^{\mathrm{TMz}}\right\rangle \gamma_{\mathrm{pq}}^{\mathrm{TM}}\left\langle\mathrm{g}_{\mathrm{pq}}^{\mathrm{TMz}} \mid \mathrm{p}_{\mathrm{kl}}\right\rangle
\end{aligned}
$$

The rectangular waveguide is characterized by its scattering operator in the modal domain by the following equation Equation 19:

$$
\begin{gathered}
\hat{\Gamma}=\sum_{\mathrm{n}=0}^{\infty} \sum_{\mathrm{m}=0}^{\infty}\left(\begin{array}{cc}
\left|\mathrm{g}_{\mathrm{nm}}^{\mathrm{TEx}}\right\rangle \gamma_{\mathrm{nm}}^{\mathrm{TE}}\left\langle\mathrm{g}_{\mathrm{nm}}^{\mathrm{TEx}}\right| & 0 \\
0 & \left|\mathrm{~g}_{\mathrm{nm}}^{\mathrm{TEy}}\right\rangle \gamma_{\mathrm{nm}}^{\mathrm{TE}}\left\langle\mathrm{g}_{\mathrm{nm}}^{\mathrm{TEy}}\right|
\end{array}\right) \\
+\sum_{\mathrm{n}=0}^{\infty} \sum_{\mathrm{m}=0}^{\infty}\left(\begin{array}{cc}
\left|\mathrm{g}_{\mathrm{nm}}^{\mathrm{TMx}}\right\rangle \gamma_{\mathrm{nm}}^{\mathrm{TM}}\left\langle\mathrm{g}_{\mathrm{nm}}^{\mathrm{TMx}}\right| & 0 \\
0 & \left|\mathrm{~g}_{\mathrm{nm}}^{\mathrm{TMy}}\right\rangle \gamma_{\mathrm{nm}}^{\mathrm{TM}}\left\langle\mathrm{g}_{\mathrm{nm}}^{\mathrm{TMy}}\right|
\end{array}\right)
\end{gathered}
$$

After determining the coefficients of diffraction in the modal and space domain, we can apply the iterative process in this example to study the phenomenon of coupling between two radiating slots. Can be modalizes this antenna structure with two operators diffraction one in the modal and space domain, as shown in the wiring diagram in Fig. 10.

Issue guides in the field are inferred from the expression of mode propagation in the spatial domain Equation 20 and 21:

$$
\begin{aligned}
& E_{\theta}=\sqrt{\frac{2}{L_{\theta} L_{Z}}} \sin \left(\frac{\pi}{L_{Z}}\left(z-\frac{L_{Z}}{2}\right)\right) \\
& E_{z}=\sqrt{\frac{2}{L_{\theta} L_{Z}}} \sin \left(\frac{\pi}{L_{\theta}}\left(r \theta-\frac{L_{\theta}}{2}\right)\right)
\end{aligned}
$$

The wave source used in the modeling, is defined in space as shown respectively (18) and (19) for both axial and radial slots Equation 22 and 23:

$$
\begin{aligned}
& A_{0}=\left(\frac{E_{z}}{\sqrt{Z_{o}}}\right) \\
& A_{0}=\left(\frac{E_{\theta}}{\sqrt{Z_{0}}}\right)
\end{aligned}
$$

Explained the system of equations in (20) is applied in the iterative solution Equation 24:

$$
\left\{\begin{array}{l}
\vec{B}=\hat{\Gamma}_{e x t} \vec{A} \\
\vec{A}=\hat{S} \vec{B}+\vec{A}_{o}
\end{array}\right.
$$


Beldi, S. et al. / American Journal of Applied Sciences 11 (8): 1426-1435, 2014

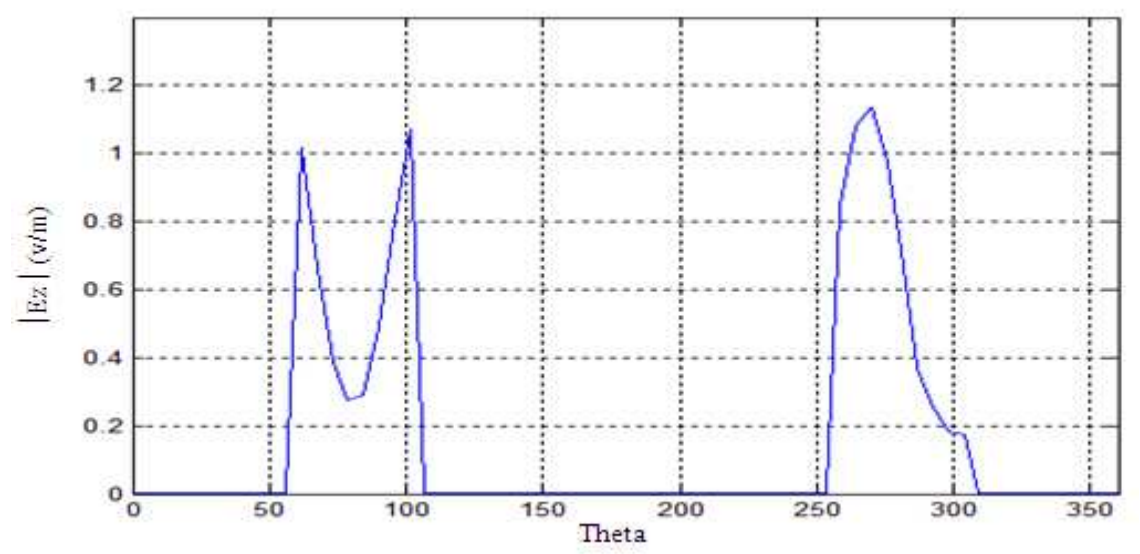

Fig. 7. Normalized Fields $|E z|$ on the surface of diffraction for: $\{\theta 1, \theta 2, \theta 3, \theta 4\}=\left\{-120,-60,60\right.$ and $\left.120^{\circ}\right\}$

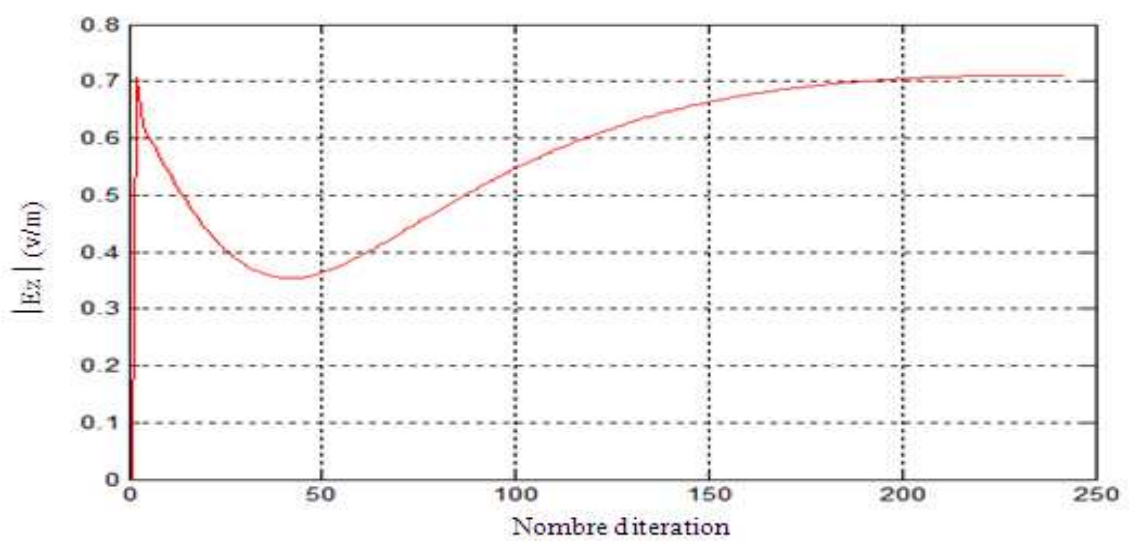

Fig. 8. Normalized Fields $\mid$ Ez $\mid$ on the surface of diffraction for: $\left\{\theta_{1}, \theta_{2}, \theta_{3}, \theta_{4}\right\}=\left\{-105^{\circ},-75^{\circ}, 75^{\circ}, 105^{\circ}\right\}$

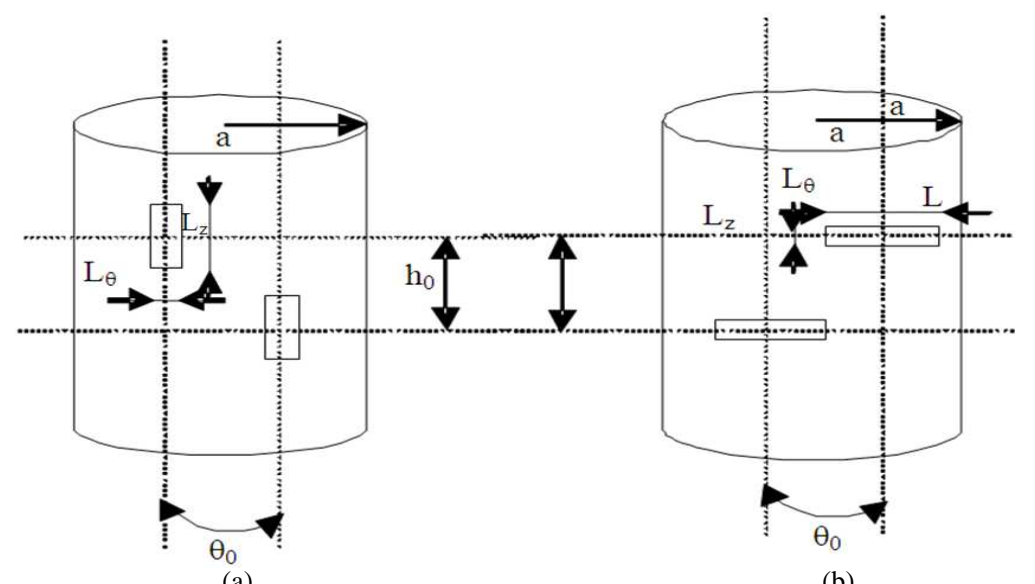

(a)

(b)

Fig. 9. Structure with: (a) Axial apertures (b) Circum ferential apertures 
Beldi, S. et al. / American Journal of Applied Sciences 11 (8): 1426-1435, 2014

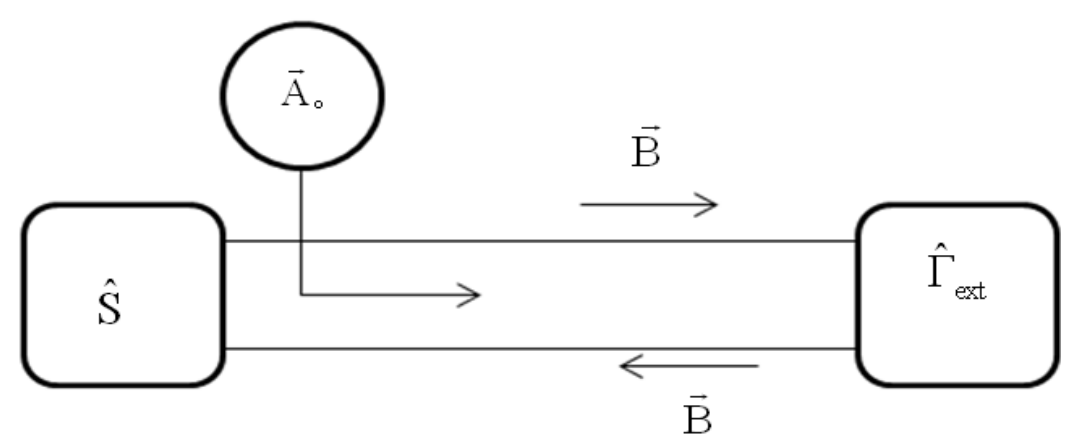

Fig. 10. Modeling the structure with the formulation on the wave

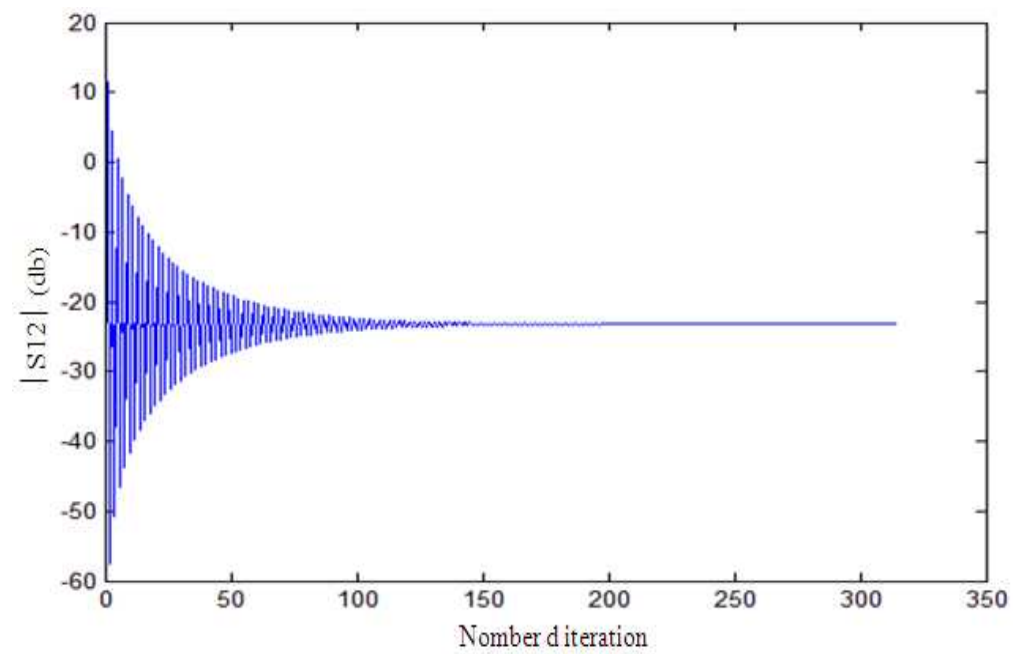

Fig. 11. Convergence curves for $|\mathrm{S} 12|$ and its mean for an angular separation of $0^{\circ}$

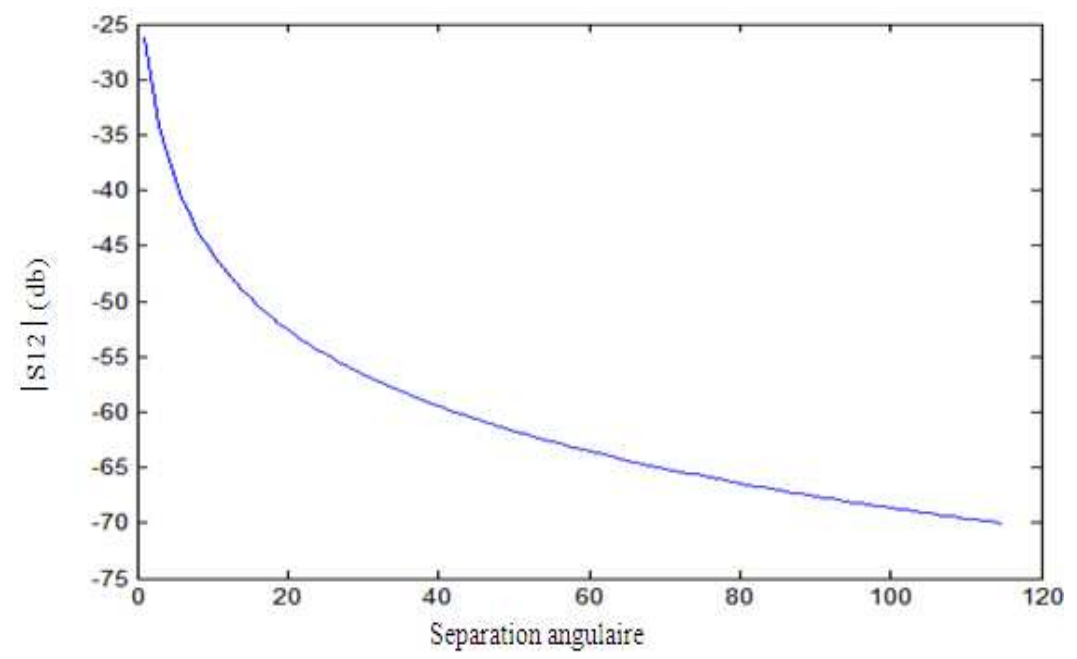

Fig. 12. Mutual coupling between axial slot antennas on a perfectly conducting cylinder, $\mathrm{z} 0=3.81 \mathrm{~cm}$ 
Beldi, S. et al. / American Journal of Applied Sciences 11 (8): 1426-1435, 2014

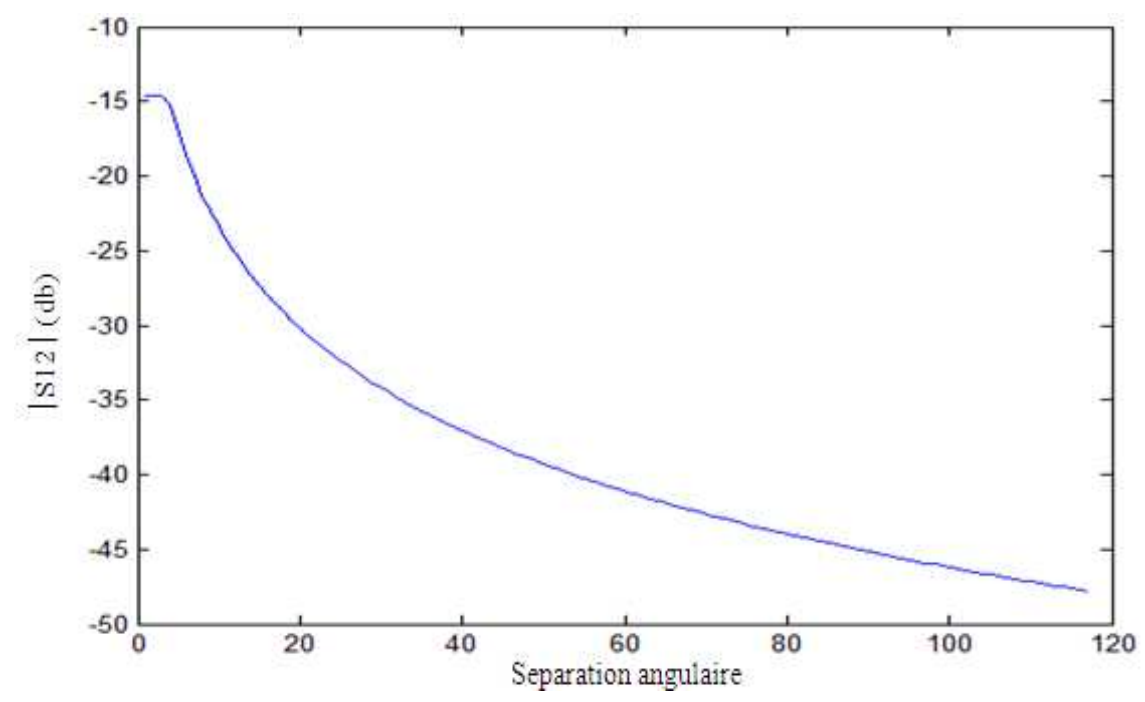

Fig. 13. Mutual coupling between circumferential slot antennas on a perfectly conducting cylinder, $\mathrm{z}_{0}=3.81 \mathrm{~cm}$

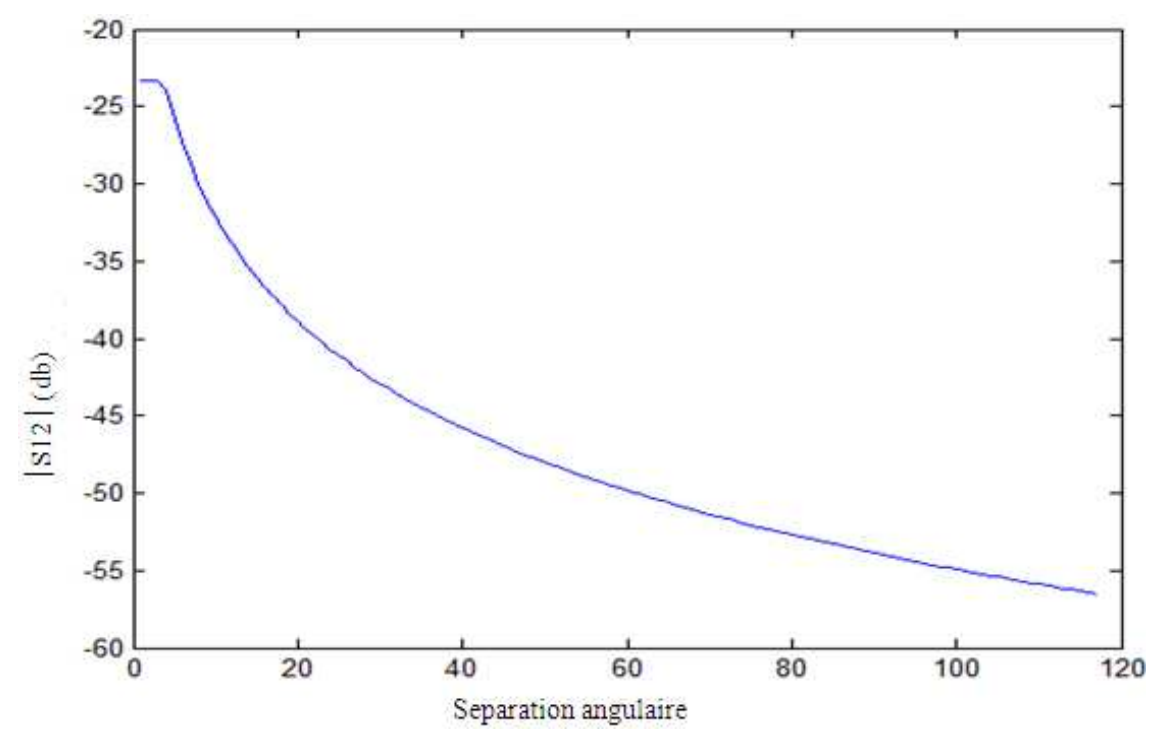

Fig.14. Mutual coupling between circumferential slot antennas on a perfectly conducting cylinder, z0 $=10.12 \mathrm{~cm}$

\subsection{Results}

After applying the concept wave in the algorithm for the iterative process, the convergence of transmission coefficient is waiting after 317 iterations shows in Fig. 11, over a distance azimuth is $h_{0}=10.16$ $\mathrm{cm}$ and an angular distance is $\theta_{0}=0^{\circ}$.

The Fig. 12 below shows the modulus of transmission coefficient $|\mathrm{S} 12| \mathrm{dB}$ depending on the angular distance $\theta_{0}$ with azimuthally distance $\mathrm{h}_{0}=3.81 \mathrm{~cm}$.
The two Fig. 13 and 14 below represent the modulus of transmission coefficient $|\mathrm{S} 12| \mathrm{dB}$ coupling between two rectangular slots for different radial distances azimuthally $h_{0}$ as a function of angular distances $\theta_{0}$.

\section{CONCLUSION}

The iterative method WCIP is applied successfully in planar structures. In this study, this method has been extended to analyze electromagnetic problems 
from the structures of cylindrical geometry. The diffraction coefficients in the modal domain and space have been explained in the cylindrical coordinate system. The method was applied to determine the diffraction on pallets cylindrical conductive. WCIP results and those previously reported in the literature are in agreement. This method seems highly appropriate to the use of planar structures, cylindrical and it can also achieve the electromagnetic problems associated with geometries of arbitrary shapes.

\section{REFERENCES}

Azizi, M.K., L. Latrach, N. Raveu, A. Gharsallah and H. Baudrand et al., 2013. A new approach of almost periodic lumped elements circuits by an iterative method using auxiliary sources. Am. J. Applied Sci., 10: 1457-1472. DOI: 10.3844/ajassp.2013.1457.1472

Baudrand, H. and S. Wane, 2009. Multiscale Circuits: Use of Auxiliary Sources. In: Passive RFIntegrated Circuits: Modelling: Characterisation Measurement, Saguet, P. (Ed.), Wiley, ISBN-10:1848211759, pp: 67-108.

Beldi, S., R. Bedira and A. Gharsallah, 2011. Coupling between two radiating apertures on a perfectly conducting cylinder. Proceedings of the 11th Mediterranean Microwave Symposium, (MMS' 11), IEEE Xplore Press, Hammamet, pp: 8-10. DOI: 10.1109/MMS.2011.6068563
Glaoui, M., H. Zairi and H. Trabelsi, 2009. Contribution to the study of the planar circuits by a hybrid method (iterative method+FDTLM method). Proceedings of the 5th International Conference: Sciences of Electronic, Technologies of Information and Telecommunications, Mar. 22-26, TUNISIA. pp: 1-4.

Harizi, H. and A. Gharsallah, 2012. Design of pass band filter in hybrid architecture planar/non-radiative dielectric waveguide integration technology. Am. J. Applied Sci., $\quad 9$ : $1538-1541$. DOI:10.3844/ajassp.2012.1538.1541

Houaneb, Z., H. Zairi, A. Gharsallah and H. Baudrand, 2011. Modeling of cylindrical resonators by waveconcept iterative process in cylindrical coordinates. Int. J. Numer. Modell., 24: 123-131. DOI: 10.1002/jnm.765

Latrach, L., N. Sboui, A. Gharsallah, A. Gharbi and H. Baudrand et al., 2009. A design and modelling ofmicrowave active screen using a combination of therectangular and periodic waveguides modes. J. Electromagnet. Waves Applic., 23: 1639-1648. DOI: $10.1163 / 156939309789476428$

Pasalic, D., J. Bornemann and R. Vahldieck, 2001. Absorbing boundary conditions in the frequencydomain TLM method and their application to planar circuits, IEEE Trans. Microwave Theory Tech., 49: 1469-1476. DOI: 10.1109/22.939928

Raveu, N., 2003. Contribution au développement de la formulation en ondes en coordonnées cylindriques: Application à l'étude d'antennes placées sur un cylindre parfaitement conducteur. Thèses, INPT, Toulouse. 